\title{
New adenovirus-based vaccine vectors targeting Pfs 25 elicit antibodies that inhibit Plasmodium falciparum transmission
}

\author{
Kathleen A. McGuire ${ }^{1,4^{*}}$, Kazutoyo Miura2 ${ }^{2}$, Christopher M. Wiethoff ${ }^{1,5}$ and Kim C. Williamson ${ }^{3}$
}

\begin{abstract}
Background: An effective malaria transmission-blocking vaccine (TBV) would be a major advance in the current efforts to eliminate and, ultimately, eradicate malaria. Antibodies against Plasmodium falciparum surface protein, Pfs25, are known to block parasite development in the mosquito vector. However, in initial clinical trials the limited immunogenicity of recombinant Pfs 25 protein-in-adjuvant vaccines has been a challenge.

Methods: Novel human adenovirus type 5 (Ad5) vectors were used in heterologous prime boost vaccination strategies to augment the immune response against Pfs25. Specifically, an Ad5 vector that directs expression of full-length, membrane-bound Pfs 25 was used as a priming immunization followed by a boost with Ad5 viral particles displaying only the Pfs 25 epitope targeted by transmission-blocking antibodies 4B7 and 1D2 (Pfs25 aa 122-134) in hypervariable region 5 of the hexon capsid protein.
\end{abstract}

Results: This heterologous prime-boost vaccine strategy induced antibodies that significantly inhibit $P$. falciparum transmission to mosquitoes in a standard membrane-feeding assay. Further, immunized mice generated a robust antiPfs 25 antibody response characterized by higher titer, higher relative avidity and a broader lgG subclass profile than observed with a homologous prime-boost with recombinant Pfs25/alum.

Conclusion: The data suggest that focusing the immune response against defined epitopes displayed on the viral capsid is an effective strategy for transmission-blocking vaccine development.

Keywords: Malaria, P. falciparum, Transmission-blocking immunity, Pfs25, Adenovirus vaccine vectors

\section{Background}

The Plasmodium parasites that cause malaria continue to be a risk for $40 \%$ of the world's population despite the introduction of artemisinin-based combination therapy and enhanced vector control measures a decade ago [1]. In addition to the approximately 200 million clinical cases a year, many infections in endemic countries are asymptomatic. Both symptomatic and asymptomatic infections produce sexual stage parasites, called gametocytes, required for malaria transmission [2-4]. This large infectious reservoir coupled with the lack of efficacy of common anti-malarials against gametocytes highlights the

\footnotetext{
*Correspondence: Kathleen.mcguire@abbvie.com

1 Department of Microbiology and Immunology, Stritch School of Medicine, Loyola University Chicago, Maywood, IL 60153, USA Full list of author information is available at the end of the article
}

need for new approaches to block the spread of the disease [5]. Mass drug administration campaigns have been shown to decrease malaria transmission in isolated areas, but are challenging to implement and even more difficult to sustain $[6,7]$. The development of a vaccine that effectively blocks malaria transmission would enhance control strategies and also provide protection against the spread of drug- or vaccine-resistant lines.

Pfs25 is currently the most advanced transmissionblocking vaccine (TBV) candidate $[8,9]$. This $25 \mathrm{kD}$ surface protein is expressed after the gametocyte is taken up in a blood meal by a mosquito and stimulated to emerge as a gamete [10-12]. Pfs25 expression continues through fertilization and differentiation into an ookinete and is thought to aid in ookinete migration across the mosquito midgut to form an oocyst [13, 14]. Pfs25-specific 
monoclonal antibodies (mAb) with potent transmissionblocking activity have been identified and the epitopes of two mAbs have been mapped to Pfs $25^{121-130}$ (ILDTSNPVKT), but this defined peptide has not been tested directly as a vaccine candidate [15-17]. To date, the recombinant protein-based vaccine candidates tested in humans show low immunogenicity; therefore, new approaches are needed to generate high titer antibody responses targeting transmission-blocking epitopes [8, 9]. In this study, Ad5-based vaccine vectors were developed to direct the Pfs25-specific antibody response to relevant, transmission-blocking epitopes.

Replication-defective adenoviruses (Ad) are an attractive vaccine platform due to their ability to potently activate the immune system $[18,19]$. In addition to their immuno stimulatory properties, Ad vectors have wellcharacterized physical properties, a relatively stable viral capsid, a genetically tractable genome that does not integrate, and can be propagated to high titer in vitro. Specifically, Ad vectors can express exogenous antigens from a transgene or can display antigens within the viral capsid itself $[19,20]$. A heterologous prime boost strategy using an Ad-pfs 25 virus followed by a modified vaccinia Ankara (MVA)-pfs25 virus that both direct secretion of the full length Pfs 25 exodomain has been shown to induce antibodies that inhibit Plasmodium falciparum transmission [21]. Recently, in an effort to enhance Adpfs25/MVA-pfs25 immunogenicity, the IMX313 peptide coding sequence was added to the $\mathrm{C}$-terminus of the Pfs25 gene in both viral vectors resulting in the secretion of a self-assembling heptamers [22].

In this study, novel Ad5-based TBVs targeting Pfs 25 were generated that induce antibodies in mice that significantly inhibit transmission of P. falciparum to Anopheles mosquitoes in a standard membrane-feeding assay (SMFA). Specifically, an Ad5 vector directing expression of full-length, membrane-bound Pfs 25 used as a priming immunization was found to generate a high titer, high relative avidity Pfs 25 antibody response with broad IgG subclasses. A boost immunization with an Ad5-vector displaying Pfs 25 transmission-blocking epitope D3 (Pfs25 aa 122-134) within the viral capsid further increased Pfs25-specific antibody titer, relative avidity, and transmission-blocking activity when compared to homologous prime-boost with alum-adjuvanted Pfs25. This approach demonstrates the efficacy of expressing a discrete epitope within the viral capsid and provides a new strategy to enhance transmission-blocking immunity.

\section{Methods}

\section{Cell lines and culture}

HeLa cells were obtained from ATCC (Manassas, VA, USA), $293 \beta 5$ cells, stably expressing $\beta 5$ integrin, were a gift from Glen Nemerow, and 293 T-REx cells were purchased from Life Technologies (Carlsbad, CA, USA). Tissue culture reagents were obtained from Mediatech (Manassas, VA, USA) and HyClone (Erie, UK). HeLa cells, 293ß5 cells and 293 T-REx cells were maintained in Dulbecco's Modified Eagle Medium (DMEM) supplemented with $1 \mathrm{mg} / \mathrm{ml}$ streptomycin, $100 \mathrm{IU} / \mathrm{ml}$ penicillin, $0.25 \mathrm{mg} / \mathrm{ml}$ amphotericin B, non-essential amino acids, $2 \mathrm{mM}$ glutamine, $10 \mathrm{mM}$ Hepes buffer, $1 \mathrm{mM}$ sodium pyruvate. 293Trex cells were maintained with $5 \mu \mathrm{g} / \mathrm{ml}$ blasticidin (Sigma Aldrich, St Louis, MO, USA). Unless specified all other reagents were from Thermo Fisher Scientific (Waltham, MA, USA).

\section{Virus generation}

Ad5gfp generation was reported before [23] and the Ad5$p f s 25$ virus was generated using a modified AdEasy system as described previously $[24,25]$. Briefly, the Pfs 25 gene (bp 1-217) was codon-optimized for expression in humans (Genscript, Piscataway, NJ, USA) and inserted it into the pshuttle-CMV vector (Agilent Technologies plasmid \#240007, Santa Clara, CA, USA). Lambda red recombineering was used to insert the pshuttle-CMV vector containing the Pfs 25 gene into the E1/E3-deleted Ad5 genome. After finding that Pfs25 expression decreased viral yield by at least tenfold, the tet operator (TO) sequence from pcDNA4/TO/myc-HisA was inserted upstream of the cytomegalovirus (CMV) promoter. Inserting TO allowed suppression of Pfs 25 expression in 293Trex cells during propagation and increased viral yield. For the Ad5-HVR-pfs25 vectors, galk recombineering with positive and negative selection steps was used as previously described [26]. Briefly, galk was amplified from the pgalk plasmid (obtained from the NCI BRB Preclinical Repository, Frederick, MD, USA) using primers containing homology to regions within the hexon capsid protein hypervariable regions 1 (HVR1) or 5 (HVR5) flanking the galk sequence to replace amino acids 145-158 and 266-278, of HVR1 or HVR5, respectively, into the in the Ad5 genome (Table 1).

Using a second round of recombineering, galk was replaced with a peptide epitope from Pfs25 epidermal growth factor (EGF) domain II, Pfs $25^{83-95}$ (referred to as D2); or domain III, Pfs $25^{122-134}$ (referred to as D3) using primer dimers of complementary oligonucleotides encoding the entire peptide sequence (flanked by sequences homologous to either HVR1 or HVR5 (Table 1). Positive recombinants were confirmed by restriction enzyme digest, PCR amplification of the $p f_{s} 25$ epitopes and sequencing of the Hexon region of the genome. The same number of amino acids were removed from the Ad5 Hexon as were inserted to conserve the size of the HVR domains. 
Table 1 Primer sequences for recombineering

\begin{tabular}{ll}
\hline Primer name & Sequence \\
\hline Primers for galk insertion & \\
HVR1 galk forward & gtgccccaatccttgcgaatgggatgaagctgctactgctcttgaaatacctgttgacaattaatcatcggca \\
HVR1 galk reverse & agaataaggcgctgcccaaatacgtgagtttttgctgctcagcttgcttcagcactgtcctgctcctt \\
HVR5 galk forward & agcaacaaaatggaaagctagaaagtcaagtggaaatgcaattttctcacctgttgacaattaatcatcggca \\
HVR5 galk reverse & gtgtctggggtttctatatctacatcttcactgtacaataccactttaggtcagcactgtcctgctcctt \\
Primers for pfs25 epitope insertion & \\
HVR1 DIII forward & gtgccccaaatccttgcgaatgggatgaagctgctactgctcttgaaataatctggatacatctaatcccgtgaagactggagtctgcagt \\
HVR1 DIII reverse & agaataaggcgcctgcccaaatacgtgagtttttgctgctcagcttgctcacaactgcagactccagtcttcacgggattagatgtatc \\
HVR5 pfsDIl forward & agcaacaaaatggaaagctagaaagtcaagtggaaatgcaattttctcaattgatgggaacccagtgtcctacgcctgcaagtgtaat \\
HVR5 pfsDII reverse & gtgtctggggtttctatatctacatcttcactgtacaataccacttaggattacacttgcaggcgtaggacactgggttcccatcaat \\
HVR5 pfsDIII forward & agcaacaaaatggaaagctagaaagtcaagtggaaatgcaattttctcactggatacatctaatcccgtgaagactggagtctgcagt \\
HVR5 pfsDIII reverse & Gtgtctggggtttctatatctacatcttcactgtacaataccactttaggacaactgcagactccagtcttcacgggattagatgtatc \\
\hline
\end{tabular}

\section{Virus preparation}

With the exception of Ad5-pfs25 (propagated in 293Trex cells) all viruses were propagated in HEK293 cells, then purified by two rounds of cesium chloride gradient centrifugation twice and dialyzed in $40 \mathrm{mM}$ Tris, $150 \mathrm{mM}$ $\mathrm{NaCl}, 10 \%$ glycerol, and $1 \mathrm{mM} \mathrm{MgCl}_{2}$ (pH 8.2) [27]. Viral concentration was determined by Bradford assay using $1 \mathrm{mg}$ of protein per $4 \times 10^{9}$ viral particles as the conversion factor (Bio-Rad Laboratories, Inc., Hercules, CA, USA). To determine viral titer, the viruses were serially diluted on HeLa cells and green fluorescent protein (GFP) expression quantified by flow cytometry. For the Ad5-pfs25 lacking the GFP reporter, the titer was determined by immunofluorescence assay (IFA) detecting adenovirus Hexon in $293 \beta 5$ cells with a dylight labelled 9C12 antibody (Developmental Studies Hybridoma Bank, University of Iowa, DSHB Product TC31-9C12.C9, Iowa City, IA, USA). For the viruses used in this study, specific infectivity ranged from 100 to 200 viral particles per GFP-transducing unit.

\section{Pfs 25 in Ad5 vectors}

To confirm Pfs 25 expression from Ad5-pfs25, HeLa cells were transfected on glass cover slips in a 24-well plate (100,000 cells per well) and $24 \mathrm{~h}$ later fixed with $3.7 \%$ paraformaldehyde-0.159 M PIPES [piperazine- $N, N^{\prime}$-bis(2ethanesulfonic acid)] buffer (Sigma) for $15 \mathrm{~min}$, blocked in phosphate buffered saline (PBS) with $10 \%$ fetal bovine serum (FBS) and $0.5 \%$ saponin (Sigma), permeabilized with $0.5 \%$ Triton X-100 (Sigma), and washed with PBS. After blocking for $1 \mathrm{~h}$ in $10 \% \mathrm{FBS}$, the cells were probed with ID2, a monoclonal antibody recognizing a conformational epitope in EGF domain III of Pfs25 [28], followed by an Alexa fluor 488-conjugated anti-mouse IgG antibody (Invitrogen, Carlsbad, CA, USA).

\section{Mice and immunizations}

All studies were reviewed and approved by the Institutional Animal Care and Use Committee of Loyola University Chicago (Maywood, IL) under IACUC protocol number 2011020. 6-12 week old male C57/BL6 wild type mice (Jackson laboratories, Bar Harbor, ME) were vaccinated intramuscularly in the left quadriceps and serum was collected 10 or 21 days following the last immunization. For protein immunizations, purified Pfs25 was adsorbed to aluminum hydroxide (Alhydrogel from Invivogen, San Diego, CA, USA) at a 1:1 ratio (in weight) of protein to alum in solution. In the first animal study, mice were immunized with $2.5 \mu \mathrm{g}$ of yPfs 25 or $10^{10}$ viral particles of Ad5-pfs 25 on day 0 and sera and splenocytes were collected on day 10 . In the second study, mice were immunized with $25 \mu \mathrm{g}$ of yPfs 25 (to increase detectable antibody titer) or $10^{10}$ viral particles of Ad5$p f s 25$, Ad5-HVR5D2, or Ad5-HVR5D3 on day 0 and sera were collected on day 21 . The third study was a primeboost vaccination study, where mice were immunized on days 0 and 21 and serum samples were collected 21 days after the boost. In the prime-boost study, protein immunizations contained $2.5 \mu \mathrm{g}$ Pfs25-alum, Ad5-pfs25 was administered at $10^{9}$ viral particles, and all Ad5-HVR$p f s 25$ vaccinations were administered at $10^{10}$ viral particles per animal.

\section{Determination of Pfs25-specific serum antibody titer and IgG subclass production}

Blood was collected via cardiac puncture and the serum was isolated, aliquoted, and stored at $-80{ }^{\circ} \mathrm{C}$ until use. Serum from each vaccination group were pooled to allow direct comparison between the enzyme-linked immunosorbent assay (ELISA)s and later SMFA. Individual ELISAs compared each vaccine group in triplicate. 
High-binding ELISA plates (Costar cat \# 07-200-35, Sigma) were coated with Pfs 25 protein at $1 \mu \mathrm{g} / \mathrm{ml}$ in coating buffer (eBioscience, San Diego, CA, USA) overnight at $4{ }^{\circ} \mathrm{C}$. The plates were then blocked with $1 \times$ assay diluent (AD) (eBioscience 00-4202-56) for $1 \mathrm{~h}$, washed with PBST (0.05\% Tween), and added serially diluted serum (in $1 \times \mathrm{AD}$ ) for $2 \mathrm{~h}$ followed by anti-mouse IgG (Fc) (Abcam, ab97265, Cambridge, MA, USA) at 1:5000 for $1 \mathrm{~h}$. 1xTMB substrate (eBioscience cat \# 00-4201-56) was added and the reaction was stopped with $1 \mathrm{M}$ sulfuric acid, before measuring the colourimetric change at $450 \mathrm{~nm}$. Antibody endpoint titers (depicted as ELISA units) were determined using an absorbance value 3 standard deviations above the PBS-immunized control mice. For the IgG subclass ELISAs all steps were performed as described above except serum samples were added at one constant dilution (1:500) and the secondary antibodies (1:500 dilution) added were specific to IgG1, IgG2a or IgG3 (Southern Biotech, cat \# 5300-05B, Birmingham, AL, USA).

\section{Determining the relative avidity of Pfs25-specific serum antibodies}

The avidity assay is a modified Pfs25-specific ELISA where plates were coated with yPfs 25 and serum was added to the wells. In this case, the plates were incubated with a fixed dilution of serum for $2 \mathrm{~h}$ at a dilution where the antibody binding reaches saturation (determined previously by assessing the O.D. for various dilutions from the antibody titer ELISA). Following serum incubation and washes, increasing concentrations of $\mathrm{NaSCN}$ were added starting from 0.25 to $4 \mathrm{M}$ diluted in PBS (Sigma) and incubated at room temperature for $15 \mathrm{~min}$ with shaking. The plate was then washed 15 times with PBST before adding the secondary antibody [anti-mouse IgG $(\mathrm{Fc})]$ and detection as previously described. For each sample, the $\mathrm{NaSCN}$ concentration $\left(\mathrm{IC}_{50}\right)$ at which the O.D. value was reduced by $50 \%$ compared to the O.D. when no $\mathrm{NaSCN}$ was added was calculated.

\section{Ex vivo splenocyte stimulation and cytokine production}

Splenocytes were harvested from mice immunized with Ad5-pfs 25 or yPfs25-alum 10 days post-immunization (the first animal study). Following red blood cell lysis, cells were added to a 96-well tissue culture plate (Costar, Sigma) with specific antigens in RPMI. Whole splenocytes were restimulated with Ad5 (10,000 particles per cell), yPfs $25(10 \mu \mathrm{g} / \mathrm{ml})$, or Concanavalin A (ConA, $2.5 \mu \mathrm{g} / \mathrm{ml}$ ) as a positive control or no antigen as the unstimulated control. After $24 \mathrm{~h}$, cell-free supernatants were collected and subjected to an ELISA to detect IFN $\gamma$ secretion following theeBioScience IFN $\gamma$ ready-set-go ELISA protocol.

\section{Generation of the Pfs 25 homology model}

The homology modeling software, SWISS-MODEL was used to generate a model of the Pfs 25 extracellular domain [29]. The primary sequence of Pfs $25^{23-217}$ was threaded onto the structure of Pvs25 [10] and further optimized through energy minimization algorithms selected by the software. Pvs 25 is $46 \%$ identical to Pfs 25 within this sequence. Disulfide bonding patterns present in the crystal structure of Pvs 25 were maintained by the Pfs25 model.

\section{Standard membrane-feeding assays (SMFAs)}

The standard membrane-feeding assay (SMFA) to assess transmission blockade has been previously described [30]. In brief, P. falciparum (NF54 strain) was cultured for 16-18 days to produce mature gametocytes in vitro. Mature gametocytes (adjusted to $\sim 0.15 \%$ of Stage V gametocytaemia) were mixed with test serum diluted 1:10 with PBS, and fed to 50 female Anopheles stephensi. Mosquitoes were kept for 8 days, and dissected $(\mathrm{n}=20$ per sample) to enumerate oocysts in their midguts. Only midguts from mosquitoes with any eggs at the time of dissection were analyzed. Percent inhibition in oocyst intensity was calculated as: $100 \times\{1-$ (mean number of oocysts in the test)/(mean number of oocysts in the control)\}.

\section{Statistics}

Splenocyte IFNy production was compared between two groups using a Student's T test. For the SMFA, the best estimates of percent inhibition, the $95 \%$ confidence interval and the $\mathrm{p}$-value of each test sample from single or multiple feeds were calculated based on the model generated in the previous study [30]. Using the same model, ratios of oocyst numbers among test groups were calculated.

\section{Results \\ Vaccination with Ad5 expressing Pfs 25 generates high titer anti-Pfs 25 antibodies consistent with $T$ cell help}

In this study, several novel Ad5-based transmission blocking vaccine vectors were generated in an effort to stimulate a high titer antibody response against Pfs25. An Ad5 vector encoding full length Pfs25 (aa 1-217) under the CMV promoter was generated first. This Pfs 25 construct includes the proposed endogenous Pfs 25 secretory signal (aa 1-21) and glycosyl-phosphatidylinositol (GPI)-anchor addition signal (aa 194-217), which directs trafficking to and retention on the plasma membrane and consequently may enhance B cell activation. After confirming the Ad5-pfs25 vector sequence, HeLa cells were transfected with the Ad5-pfs 25 genome and $24 \mathrm{~h}$ post-transfection the majority of Pfs 25 concentrated at 
the periphery of the cells using Pfs25-specific, transmission-blocking monoclonal ID2 suggestive of cell surface localization [28] (Fig. 1). Mice were immunized with Ad5-pfs 25 ( $10^{10}$ viral particles) or a current transmission blocking vaccine candidate, yPfs $25(2.5 \mu \mathrm{g})$ adsorbed to aluminum hydroxide (yPfs25-alum) and the Pfs25 immune response was compared. yPfs25-specific antibodies were detected 10 days post-immunization with Ad5-pfs25 (Fig. 2a). Splenocytes harvested 10-days post Ad5-pfs25 vaccination exhibited a significant increase in adenovirus-specific IFNY secretion following restimulation with Ad5-pfs25 but not following yPfs25 stimulation (Fig. 2b).These data suggest Ad5-pfs25-immunized mice generated Ad-specific T cells, which may help B cell responses to Pfs 25 (Fig. 2b). In contrast, splenocytes from yPfs25-alum-immunized mice did not exhibit IFN $\gamma$ production upon Ad5-pfs25 stimulation ex vivo (Fig. 2b) and neither immunization elicited detectable Pfs25-specific $\mathrm{T}$ cell responses as assessed by monitoring IFNy (Fig. 2b).
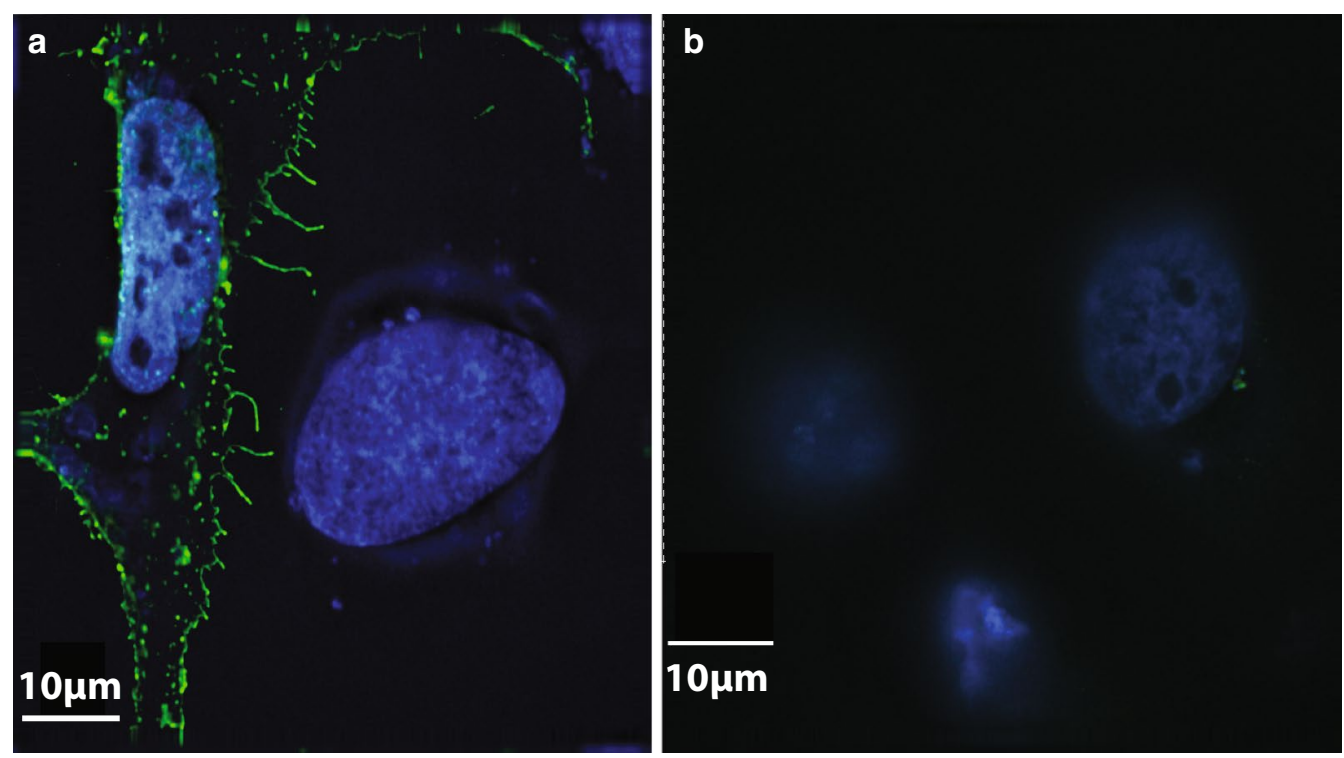

Fig. 1 Pfs 25 expression on Hela cells. Hela cells were transfected with an Ad5 shuttle vector with (a) or without (b) the codon-optimized Pfs 25 gene under the control of the CMV immediate early promotor. Twenty-four hours post transfection, cells were fixed, permeabilized, and probed with ID2, a conformation-dependent Pfs25-specific monoclonal antibody, then probed with an Alexa Fluor488-conjugated anti-mouse secondary antibody and visualized by IFA
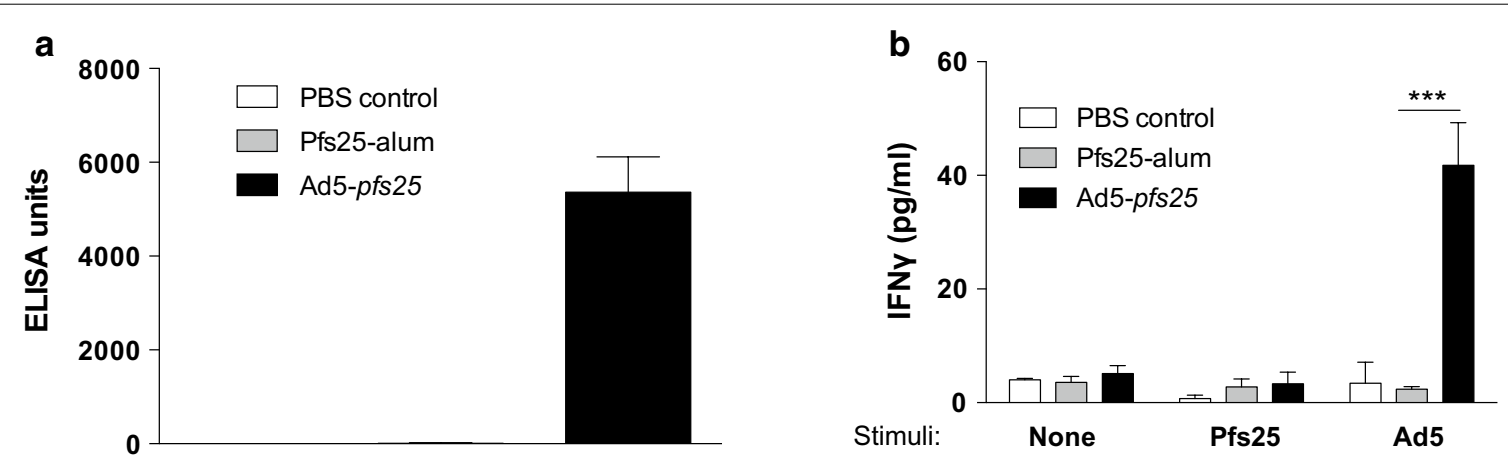

Fig. 2 Pfs 25 specific lg production and T-cell activation following Ad5-pfs 25 primary vaccination. Mice ( $n=3$ per group) were immunized with Ad5 expressing Pfs 25 ( $10^{10}$ particles) or recombinant yPfs 25 -alum ( $2.5 \mu \mathrm{g} /$ mouse) on day 0 , and serum and splenocyte samples were collected on day 10. a yPfs25-specific ELISA, yPfs25-specific ELISA were performed and data depict pooled serum from 3 animals per group tested in triplicate in 3 independent ELISAs. The average ELISA unit and standard deviation are plotted. b Splenocyte IFNy secretion. Splenocytes $(n=3$, tested individually) were incubated without or with yPfs 25 at $10 \mu \mathrm{g} / \mathrm{ml}$ or Ad5 at 10,000 particles per cell for $24 \mathrm{~h}$ as indicated on the $x$-axis. IFNy secretion was detected by ELISA and the average and standard deviation are plotted. Statistical significance was determined by Student's T test; ${ }^{* * *} p<0.001$ 
Primary Ad5-pfs25 vaccination generates a high avidity Ig response with a broad IgG subclass profile

To further characterize the Pfs25-specific antibody response, a second immunization study was conducted and assessed the relative avidity and IgG subtype profile following vaccination at the peak of the antibody response 21 days post-immunization. In this regimen the dose of yPfs 25 protein was increased tenfold to $25 \mu \mathrm{g}$ to generate a detectable Pfs25-specific antibody response to compare with Ad5-pfs25 immunization. Under these conditions 21 days post-immunization, both vaccinations elicit anti-yPfs 25 antibody titers (Fig. 3a). Antibodies elicited by Ad5-pfs 25 had a higher relative avidity to yPfs 25 than those induced by yPfs 25 /alum vaccination as demonstrated by their fourfold greater $\mathrm{IC}_{50}$ (Fig. 3b). In addition to higher relative avidity, Ad5-pfs 25 vaccination generated a broader array of IgG subclasses (Fig. 3c, d), while yPfs25-alum primarily induced anti-yPfs 25 IgG1 titers, which is not unexpected from an alum-adjuvanted protein vaccine.
Heterologous prime-boost vaccination enhances the Pfs25-specific antibody response

To examine the effect of a prime-boost vaccination strategy, a third immunization study was performed. Ad5pfs 25 was used as the priming immunization followed by either yPfs 25 or 2 novel Ad5-vectors expressing putative Pfs25 transmission-blocking epitope D2 (Pfs25 ${ }^{83-95}$ ) or D3 (Pfs $\left.25^{122-134}\right)$ designed to focus the immune response toward these regions within EGF-like domains II and III of Pfs25. A group of mice were immunized with yPfs25-alum (homologous prime-boost) as a comparator group. Peptide D2 is located in EGF domain II which was found to be the target of transmission-blocking antibodies induced in rabbits after yPfs25 immunization, while domain D3 is located in EGF domain III and corresponds to the epitope for the Pfs25-specific transmission-blocking mAb, 4B7 (Fig. 4b) [15-17]. Based on the crystal structure of the Plasmodium vivax Pfs25 paralog, Pvs25, both domains D2 and D3 are located on exposed loops in the EGF domains (Fig. 4b) [10]. Ad5 constructs
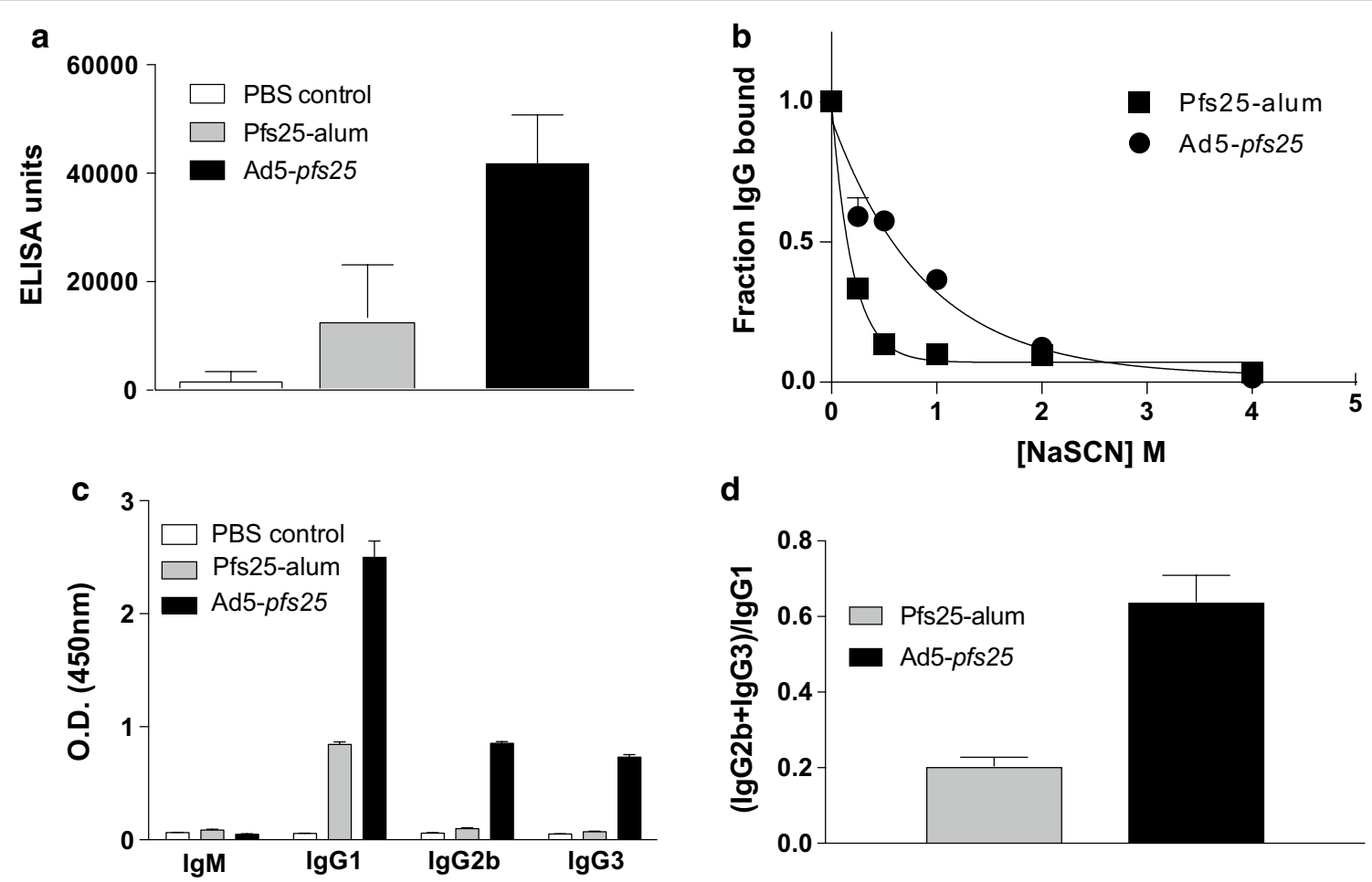

d

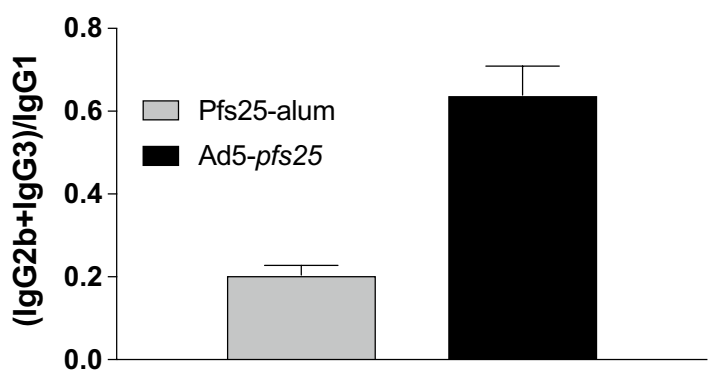

Fig. 3 Pfs25-specific serum antibody response after primary immunization with an Ad vector expressing Pfs25. Mice $(n=3$ per group) were immunized with Ad5 expressing Pfs 25 ( $10^{10}$ particles) or recombinant yPfs 25 -alum ( $25 \mu \mathrm{g} / \mathrm{mouse}$ ) on day 0 , and serum samples were collected on day 21. a yPfs25-specific antibody titer. yPfs25-specific ELISA was performed and data depict pooled serum from 3 animals per group tested in triplicate in 3 independent ELISAs. The average ELISA unit and standard deviation are plotted. b yPfs25-specific avidity. Sera were added to a yPfs 25 -coated ELISA plate at a single dilution and then incubated with increasing concentrations of NaSCN. The remaining yPfs 25 -specific antibodies were detected with an HRP-conjugated anti-mouse lgG (Fc). The fraction of Ig bound was determined by dividing the optical density (OD) at each NaSCN concentration by the OD in the absence of NaSCN and the nonlinear best-fit line was plotted for each data set. $\mathbf{c}$, $\mathbf{d}$ yPfs 25 -specific lgM and lgG subclass profile. yPfs25-specific ELISA was performed and the yPfs25-specific IgM and IgG subclasses were detected with HRP-conjugated specific antibodies. The average OD and standard deviation for each isotype/subclass (c) and the ratio of the O.D. for the $\operatorname{lgG} 1$ to the O.D.s of $\operatorname{lgG} 2 \mathrm{~b}$ and $\operatorname{lgG} 3$ (d) are shown 
a
Sec: 1) MNKLYSLFLFLFIQLSIKYN
EGFI: 21) NAKVTVDTVCKRGFLIQMSGHLECKCENDLVLVNEETC
EGFII: 59) EEKVLKCDEKTVNKPCGDFSKCIKIDGNPVSYACKCNLGYDMVNNVC
EGFIII: 106) IPNECKNVTCGNGKCILDTSNPVKTGVCSCNIGKVPNVQDQNKC EGFIV: 150) SKDGETKCSLKCLKENETCKAVDGIYKCDCKDGFIIDNESSICTAFS GPI: 197) AYNILNLSIMFILF SVCFFIM
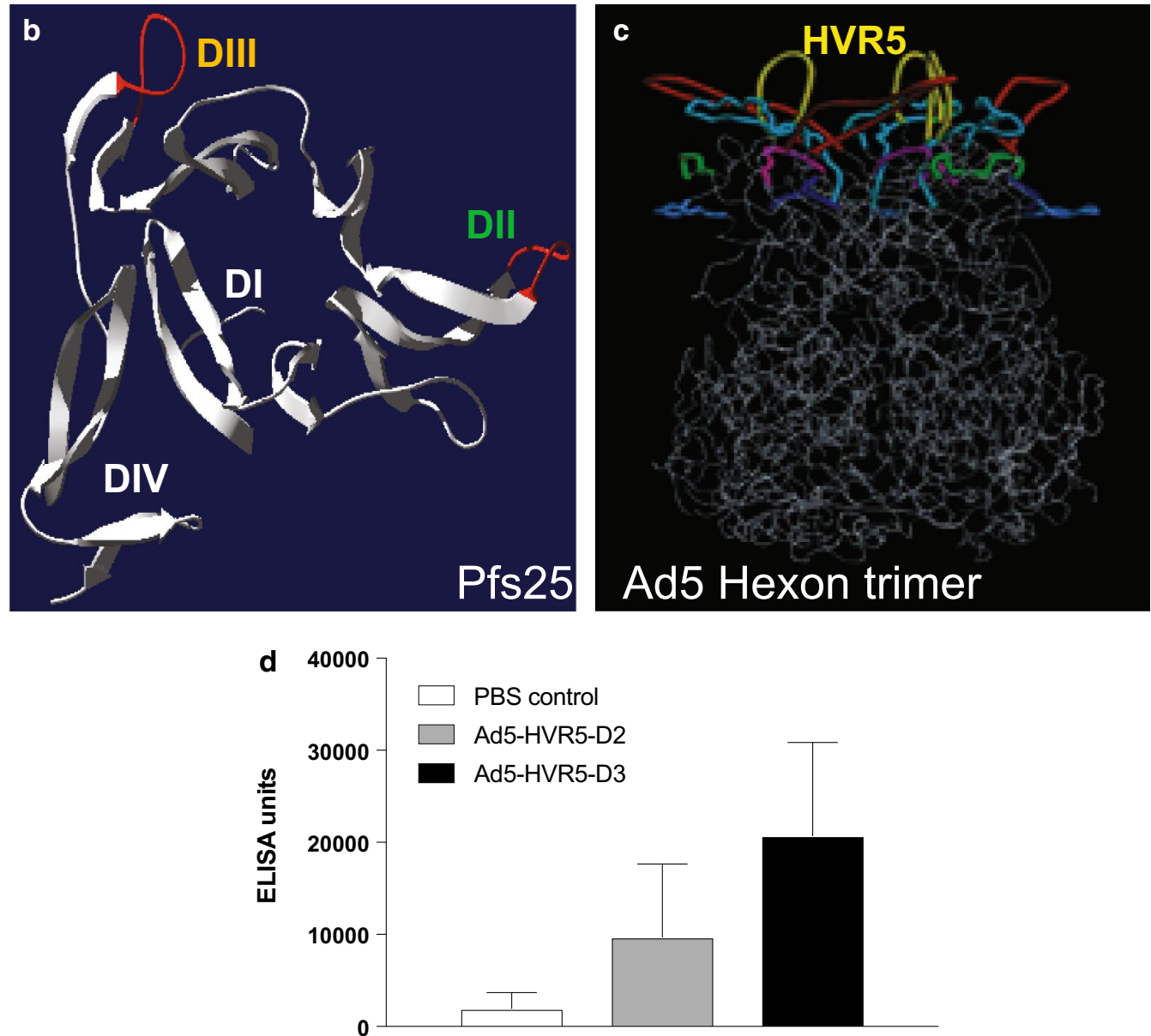

Fig. 4 Pfs 25 epitope specific vectors design. a Amino acid sequence of Pfs25. The selected Pfs25 EGF domains II and III from which the epitope peptides (denoted as D2 and D3) were chosen for HVR insertion are indicated in green and orange, respectively. b Predicted Pfs 25 structure based on the structure of the P. vivax homolog, Pvs25. The D2 and D3 epitopes in EGF domains II and III, respectively, are highlighted in red. c Ad5 Hexon capsid protein structure. Ad5 Hexon trimer structure (Protein Data Bank 1P30) with the modelled HVRs highlighted (HVR1, red; HVR2, green; HVR3, pink; HVR4, light blue; HVR5, yellow; HVR6, blue; HVR7, cyan). d yPfs25-specific antibody titer. Mice ( $\mathrm{n}=3$ per group) were immunized with $10^{10}$ viral particles of Ad5 displaying the D2 or D3 Pfs25 peptide epitope within HVR5 (denoted Ad5-HVR5D2 or Ad5-HVR5D3, respectively) or PBS control and yPfs25-specific ELISA was performed and data depict endpoint dilutions for 3 individual animals where error bars depict standard deviation

were designed to display either D2 or D3 in hypervariable regions (HVR) 5 or 1 of the hexon capsid protein (Fig. 4c) resulting in the production of a virion displaying theoretically 720 copies of the inserted Pfs 25 domain on its surface. Several studies demonstrate that HVR-modified virions maintain proper viral assembly, structure and infectivity and elicit a response to the antigen insertion [31, 32]. While insertion of Pfs25 epitopes into either HVR did not affect virus assembly, as determined during purification of the viral vectors, insertion into HVR1 decreased vector specific infectivity tenfold and therefore are not included in further analysis. In contrast, 
insertion into HVR5 had no negative effects on infectivity. A single immunization with either Ad5-HVR5D2 or Ad5-HVR5D3 induced antibodies that recognized yPfs25 recombinant protein in an ELISA assay indicating that the Pfs25 D2 or D3 epitopes were displayed on the virion and immunogenic (Fig. 4d).

In mice primed with Ad5-pfs25, a secondary immunization with Ad5-HVR5D2, Ad5-HVR5D3, or yPfs25alum generated higher anti-yPfs25 IgG ELISA titers as compared to our benchmark, yPfs25-alum homologous prime-boost (Fig. 5a, b). Sera obtained after the heterologous prime-boosts also had greater relative avidity than serum obtained after homologous prime-boost with yPfs25-alum (Fig. 5c) or a single Ad5-pfs 25 immunization (Fig. $3 \mathrm{~b}$ ) as indicated by a nearly threefold higher $\mathrm{IC}_{50}$. Upon examining IgG subclass following prime-boost immunizations the homologous prime-boost with yPfs25-alum was found to generate an IgG1-dominated response (Fig. 5d, e) as reported previously [22, 33]. Of note, the Ad5-pfs 25 prime and yPfs 25 boost group showed similar avidity and IgG subclass profiles as the Ad5-pfs25 prime and Ad5-HVRD2 or D3 boost groups, rather than the yPfs25-alum homologous prime-boost group (Fig. 5c). The results suggest the Ad5-pfs25 primary immunization determined the responses.

\section{Heterologous prime-boost vaccination with Ad5-based vectors blocks $P$. falciparum transmission to the mosquito vector}

Although the heterologous prime boost vaccines enhanced the yPfs 25 immune response, the critical question remaining is whether transmission-blocking efficacy
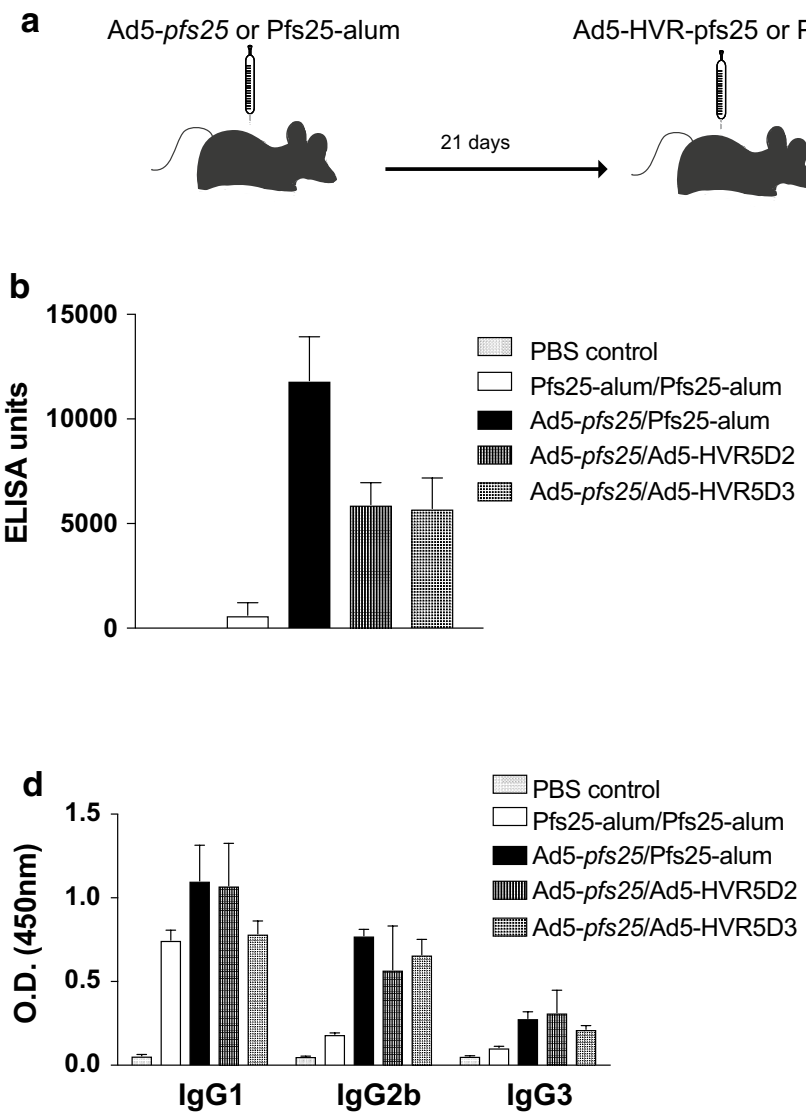

e
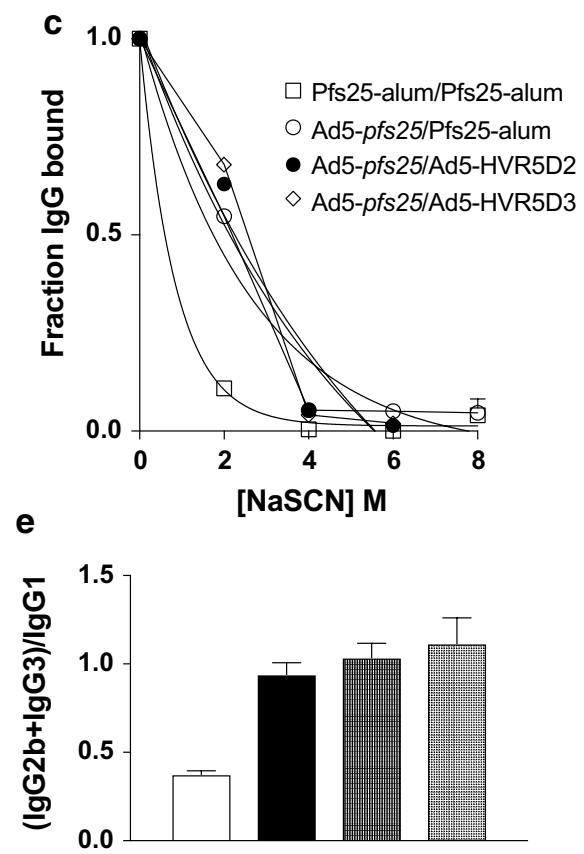

Pfs25-alum/Pfs25-alum m mIIIII Ad5-pfs25/Ad5-HVR5D2 Ad5-pfs25/Pfs25-alum A Ad5-pfs25/Ad5-HVR5D3

Fig. 5 Pfs25-specific serum antibody response upon heterologous prime-boost vaccination. a Vaccination schematic. Mice ( $n=6$ per group) were immunized with yPfs 25 -alum ( $2.5 \mu \mathrm{g} /$ mouse) or Ad5 vector Pfs 25 ( $10^{9}$ particles) on day 0 , and followed by boost immunization with yPfs 25 -alum ( $2.5 \mathrm{\mu g}$ /mouse) or the different Ad5-HVR-pfs 25 vectors ( $10^{10}$ particles). The serum samples were collected 21 days after the boost. b yPfs 25 -specific antibody titer. yPfs25-specific ELISA was performed and data depict pooled serum from 6 animals per group tested in triplicate in 3 independent ELISAs. The average ELISA unit and standard deviation are plotted. c yPfs25-specific antibody avidity. The assay was performed as described for Fig. 3. The fraction of Ig bound was determined by dividing the OD at each NaSCN concentration by the OD in the absence of NaSCN and the nonlinear best-fit line was plotted for each data set. d, e yPfs25-specific lgG sublass profile. The assay was performed as described for Fig. 3. The average OD and standard deviation for each isotype/subclass (d) and the ratio of the O.D. for the $\lg G 1$ to the O.D.s of $\lg$ G2b and $\operatorname{lgG} 3$ (e) are shown. Data depict the ratio of the OD for $\lg G 1$ to the ODs of $\lg G 2 \mathrm{~b}$ and $\lg G 3$ 
increases. Therefore, transmission-blocking activity was tested by SMFA using An. stephensi mosquitoes [30]. Consistent with the enhanced humoral response, all the Ad5-pfs25 prime-heterologous boost vaccines tested induced serum (tested at 1:10 dilution) that significantly reduced parasite load compared to the yPfs25-alumimmunized sera (Fig. 6). Serum harvested 21 days after the Ad5-pfs25 prime Ad5-HVR5D3 or yPfs25-alum boost significantly reduced the average oocyst number per mosquito midgut by $82.5 \%$ (the best estimate from 3 independent SMFAs, and the 95\% confidence interval $(95 \% \mathrm{CI})$ was $67.3-90.7, \mathrm{p}<0.001)$ and $78.1 \%(95 \%$ CI 58.6-88.7; $\mathrm{p}<0.001$ ), respectively. Serum from the Ad5-pfs25 prime Ad5-HVR5D2 boost also significantly reduced transmission, but the inhibition level was lower (52.6\% inhibition; 95\% CI 13.7-74.9; $\mathrm{p}=0.023$ ) and more variable between SMFAs. In contrast, homologous prime-boost with the same dose of yPfs25-alum did not

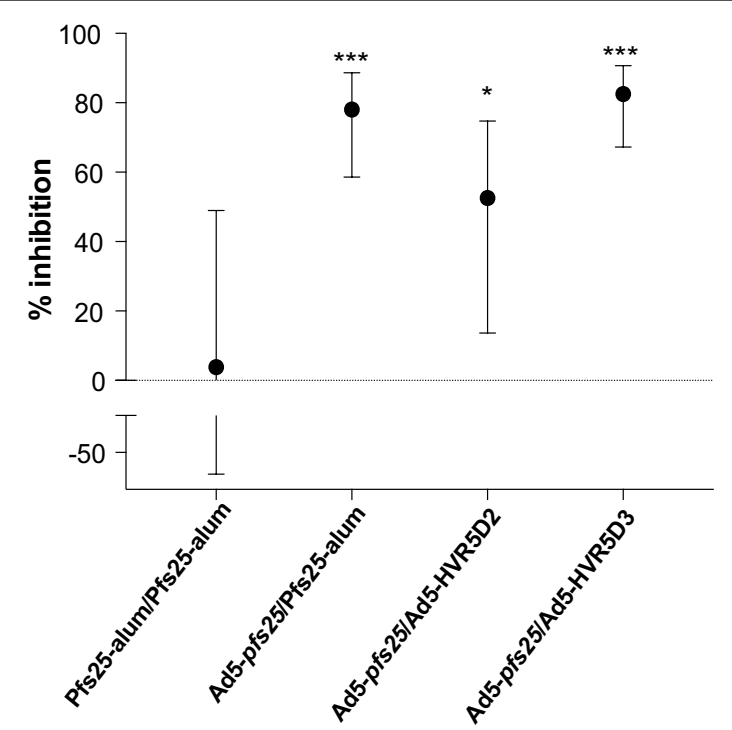

Fig. 6 Vaccination induced transmission-blocking activity. Two independent sets of mice were immunized with the test groups, yPfs 25 homologous prime boost (yPfs25-alum/yPfs25-alum) or the 3 heterologous prime boost strategies (Ad5-pfs25/yPfs25-alum, Ad5-pfs25/Ad5-HVR5D2, and Ad5-pfs25/Ad5-HVR5D3) as described in "Methods". Serum was pooled from the test groups from each immunization ( $n=3$ mice for all the immunization groups except the 2nd yPfs 25 homologous prime boost immunization, which included 6 mice) and tested in SMFAs at a 1:10 dilution. Pooled serum from mice immunized with an Ad5-luciferase vector was the negative control in each assay. The first SMFA tested pooled serum from the first immunization, while the second and third SMFAs tested pooled serum from the second immunization. All three SMFAs were included in the statistical analysis. The best estimate of the percent inhibition from 3 independent SMFAs is plotted with the 95\% confidence interval for each sample calculated using a zero-inflated negative binomial model [30] (*** $<0.001,{ }^{*} p<0.05$ as compared to baseline) significantly reduce transmission (3.8\% inhibition; $95 \%$ $\mathrm{CI}-67.5$ to $48.9 ; \mathrm{p}=0.921)$. The differences in oocyst numbers between homologous prime-boost with yPfs 25 alum and all the Ad5-pfs25 prime-heterologous boost vaccines were significant $(\mathrm{p}<0.024)$.

\section{Discussion}

Transmission-blocking vaccines employing Pfs25 show promising preclinical results, although effective vaccine strategies in humans remain a work in progress. In this study, new Ad5 virus-pfs 25 vectors were developed in an effort to enhance the induction of antiserum that significantly reduces malaria transmission in a SMFA. Specifically, an initial priming immunization with an Ad5-pfs25 vector that directs expression of membrane-bound Pfs 25 followed by a booster injection of Ad5 virions displaying 720 copies of a single, defined Pfs 25 transmissionblocking epitope (D3) in the HVR5 of the hexon capsid protein induce antibodies that significantly inhibit $P$. falciparum oocyst production. Recombinant yPfs25 could also effectively boost the initial Ad5-pfs 25 immunization and generate antibodies that significantly reduce transmission in an SMFA. In marked contrast, serum obtained after homologous prime/boost immunizations using the same dose of recombinant yPfs 25 in alum did not effectively reduce oocyst production. This differential response against yPfs 25 following a homologous yPfs 25 or heterologous Ad5-pfs25 immunization demonstrates the key role of Ad5-pfs 25 in priming the immune response for the production of high avidity, high titer anti-Pfs 25 antibodies. This enhanced immune response is likely due to the ability of Ad5-pfs 25 to stimulate a T cell response, which was not observed following immunization with recombinant yPfs 25 in alum [34]. Previously, investigators have shown that a prime boost strategy using 2 distinct viral vectors, an adenovirus isolated from chimpanzees (ChAd63) followed by a modified Vaccinia virus (MVA), both directing expression of secreted, monomeric Pfs25, also induced transmission reducing antibodies [21]. However, this vaccination strategy generated relatively modest anti-Pfs 25 antibody titers prompting the additional development of other Pfs25-targeting vaccines including Pfs25IMX313. Pfs25-IMX313 is a secreted chimeric protein made up of Pfs 25 fused to the self-associating oligomerization domain of the chicken complement inhibitor C4bbinding protein [22]. The addition of IMX313 Pfs 25 to both ChAD63 and MVA Pfs25 $5^{22-193}$ expression vectors enhanced immunogenicity and also improved the ability of the antiserum to block oocyst production as compared to ChAD/MVA-pfs25 vectors without IMX313 or recombinant Pfs25-IMX313 protein. These results suggest roles for both multimerization and the viral vector in enhancing the antibody response. 
This work extends the current Ad-based vector immunization strategies to evaluate Ad viral vectors that direct membrane expression of Pfs 25 and focus the immune response to proposed transmission-blocking epitopes in Pfs25. To target Pfs25 expression to the surface of the cell, the Ad5-pfs25 vector, which includes the full-length Pfs 25 gene containing the both the secretory and GPI anchor signal sequence of Pfs 25 , was developed. These changes to the Ad-based Pfs 25 expression vector resulted in multiple copies of Pfs 25 expressed on the surface of the virally transduced cell [35]. Expression of membranebound Pfs 25 is likely to improve antigen persistence and lower antigen clearance, which can be a problem for soluble antigens. Furthermore, surface expression of fulllength Pfs 25 on a single cell could allow the display of antigen for direct $B$ cell recognition in close proximity to processed adenovirus antigen in the context of MHC to activate $\mathrm{T}$ cells, thus providing the requisite $\mathrm{T}$ cell help to enhance B cell activation. The high anti-yPfs 25 antibody titers obtained 10 days post-immunization with Ad5-pfs 25 compared to titers following yPfs25/alum as well as the concurrent isolation of T-cells that produced IFNY in response to restimulation with Ad5 is consistent with the dual activation of $\mathrm{B}$ and $\mathrm{T}$ cells. Previous work demonstrated that Ad vector-directed antigen expression induces significantly higher antibody titers than titers generated following co-immunization of an empty Ad vector and recombinant protein [36]. From this experiment the authors suggested that expression of a target antigen in the context of Ad5 transduction improves antibody titer and likely does so by providing $\mathrm{T}$ cell help in the lymph node follicle [36].

Next, to focus the immune response to specific Pfs 25 epitopes Ad5-based vectors displaying Pfs25 epitopes within hypervariable regions (HVR) 1 or 5 of the hexon capsid protein on the viral vector surface (Ad5-HVRD2 or D3) were generated for use as booster immunizations following a prime with Ad5- $p f s 25$. Other studies demonstrate that displaying antigen epitopes within these HVR domains of the viral capsid elicit antibodies to the target antigen [32, 37-39]. By expressing these Pfs 25 epitopes within Hexon, which is the major viral capsid protein, 720 copies of the Pfs 25 epitopes decorate the Ad5-HVRpfs25 vector surface. Pfs25 epitopes (D2 and D3) from EGF domains DII and DIII, which have been implicated as the targets of transmission-blocking antibodies, were selected to display in Hexon HVRs 1 or 5 [16]. These viral vectors elicited high titer and high avidity anti-yPfs 25 antibodies with similar IgG subclass profiles. However, the reduction in oocysts using serum obtained from mice boosted with Ad5-HVR5D2 was lower. The 12-amino acid D2 peptide was selected based on in silico homology with the Pvs 25 structure and, perhaps, in Pfs 25 is a slightly different epitope localizes to the loop of the EGF domain 2 [10, 40-42]. Further, a larger region of DII may need to be included in the $\mathrm{D} 2$ peptide since, in the original study implicating EGF domain II as a transmissionblocking target, domain II included a larger region of Pfs25 (amino acids 59-110) expressed in yeast [16]. By itself the recombinant Pfs 25 domain II was poorly immunogenic, but a booster vaccination with a recombinant protein corresponding to the full exodomain of recombinant Pfs 25 successfully induced transmission-reducing antibodies. It is possible that additional boosts with a larger region of Pfs 25 would have increased the response to Ad5-HVR5D2. Indeed, serum obtained after boosting with recombinant yPfs 25 in alum following a heterologous Ad5-pfs 25 priming immunization inhibited oocyst production as effectively as boosting with Ad5-HVR5D3. This finding is in marked contrast to the ineffectiveness of a homologous yPfs25/alum prime/boost immunization strategy, even though the same recombinant yPfs 25 was used. These data strongly support the role of the Ad5 viral vector in enhancing the primary immune response and is the first demonstration of an Ad vector prime, recombinant protein boost strategy for Pfs25, although this has been suggested in the discussion of previous vaccine studies [43]. The success of this approach shows the promise of a strategy that combines the enhanced $\mathrm{T}$ cell activation of an Ad vector priming immunization with boosts using a recombinant protein vaccine [21, 44]. Future studies could include the evaluation of the longevity of the response and optimization of the viral vector and dosing schedule.

This study has highlighted the role for the D3 epitope as a transmission-blocking candidate since a booster immunization with Ad-HVR5D3, which displays only the 13 amino acid D3 peptide on the viral capsid, generates a transmission-reducing antibody response that is comparable to a booster immunization with the entire recombinant yPfs 25 protein. The efficacy of this domain is consistent with previous findings demonstrating recognition of the D3 domain by two different Pfs 25 specific transmission-blocking antibodies 4B7 and 1D2 [15-17]. However, the previous attempt to induce transmissionblocking antibodies using yeast-produced recombinant protein corresponding to the entire Pfs 25 EGF domain III (amino acids 107-156) was not effective [16]. It is possible that limiting the domain to just the transmission-blocking epitope could serve to focus the response. Moreover, incorporating the peptide within the viral capsid allows for stronger signaling through interactions between the multiple Pfs 25 epitopes displayed on the capsid and multiple BCRs, as well as taking advantage of the immuno stimulatory properties of the Ad vector. These Ad5-pfs25 prime and Ad5-HVR capsid display 
vectors could also be used to screen additional Pfs 25 peptides or a peptide library for transmission-blocking epitopes. Purified Ad5-HVR-Pfs25 peptide virions could be directly screened for recognition by transmissionblocking monoclonal antibodies or the ability to deplete transmission-blocking antibodies from serum. Additionally, Ad5-HVR-Pfs 25 peptide antiserum could be tested for gamete surface recognition or transmission-blocking activity by SMFA. Taken together, these approaches could efficiently identify TBV epitopes that could be directly tested alone or in combination for the ability to induce malaria transmission-blocking antibodies.

\section{Conclusion}

This study has identified several new Ad5-based Pfs25 vectors to enhance the humoral immune response to $\mathrm{Pfs} 25$. Compared to the recombinant yPfs25-alum vaccine, Ad5-pfs 25 elicited high Pfs25-specific IgG titers with greater transmission-blocking activity making Ad vectors a superior method for primary immunization. This observed improvement in the anti-Pfs 25 response may be due to the Ad5-specific $\mathrm{T}$ cell response induced by Ad5-pfs 25 vaccination. This robust primary immune response mediated by Ad5-pfs25 was exploited by combining it with boost vaccinations using Ad-based vectors that display relevant, transmission-blocking Pfs 25 epitopes on its surface (Ad5-HVR5D3 or D2). The heterologous prime-boost approach increased the Pfs25-specific antibody titer, relative avidity, and transmission-reducing activity as compared to homologous prime-boost with Pfs25-alum. These novel transmissionblocking vaccine vectors provide approaches to augment and focus anti-Pfs 25 immunity.

\footnotetext{
Abbreviations

Ad5: adenovirus type 5; ChAd63: adenovirus isolated from chimpanzees; AD: $1 \times$ assay diluent; ConA: Concanavalin A; CMV: cytomegalovirus; DMEM: Dulbecco's Modified Eagle Medium; ELISA: enzyme-linked immunosorbent assay; EGF: epidermal growth factor; FBS fetal bovine serum GPI: glyceryl-phosphatidylinositol; GFP: green fluorescent protein; HVR: hexon hypervariable regions; IFNY: interferon gamma; IFA: immunofluorescence assay; Ig: immunoglobulin molecule; MVA: modified Vaccinia virus; mAb: monoclonal antibody; OD: optical density; D2: Pfs 25 amino acids 83-95; D3: Pfs 25 amino acids 122-134; PBS: phosphate buffered saline; SMFA: standard membrane-feeding assay; TO: tet operator; TBV: transmission-blocking vaccine; yPfs25: yeast-produced recombinant Pfs 25 .
}

\section{Authors' contributions}

KAM and CMW conceived of the project and designed the experiments. KAM performed the experiments. KM performed the SMFA experiments. KM, CMW and KW supervised the experiments and all authors contributed to the data analysis and wrote the manuscript. All authors read and approved the final manuscript.

\section{Author details}

${ }^{1}$ Department of Microbiology and Immunology, Stritch School of Medicine, Loyola University Chicago, Maywood, IL 60153, USA. ${ }^{2}$ Laboratory of Malaria and Vector Research, National Institute of Allergy and Infectious Diseases,
National Institutes of Health, Rockville, MD, USA. ${ }^{3}$ Department of Microbiology and Immunology, Uniformed Services University, Bethesda, MD 20814 USA. ${ }^{4}$ Present Address: Abbvie, 1 North Waukegan Road, North Chicago, IL 60064, USA. ${ }^{5}$ Eli Lilly and Company, Lilly Corporate Center, Indianapolis, IN 46285, USA.

\section{Acknowledgements}

The authors thank David Narum (NIH) for providing the yPfs 25 protein and Carole Long for critical reading of the manuscript.

\section{Competing interests}

The authors declare that they have no competing interests.

Availability of data and materials

All relevant data are within the paper.

Consent for publication

All authors read and approved to its final submission.

\section{Ethics approval}

All studies were reviewed and approved by the Institutional Animal Care and Use Committee of Loyola University Chicago (Maywood, IL) under IACUC protocol number 2011020

\section{Funding}

This work was supported by grants from the National Institutes of Health (T32Al007508-14 to KAM) and The Arthur J. Schmitt Predoctoral Fellowship to KAM. In addition, the SMFA was supported by the intramural program of the National Institute of Allergy and Infectious Disease/NIH and by the PATH Malaria Vaccine Initiative. The authors have no financial interest to disclose.

\section{Publisher's Note}

Springer Nature remains neutral with regard to jurisdictional claims in published maps and institutional affiliations.

Received: 29 November 2016 Accepted: 6 June 2017

Published online: 15 June 2017

\section{References}

1. WHO. World malaria report. Geneva: World Health Organization; 2015. http://www.who.int/malaria/publications/world-malaria-report-2015/ report/en/. Accessed 11 Oct 2013.

2. Imwong M, Stepniewska K, Tripura R, Peto TJ, Lwin KM, Vihokhern B, et al. Numerical distributions of parasite densities during asymptomatic malaria. J Infect Dis. 2016;213:1322-9.

3. Hofmann N, Mwingira F, Shekalaghe S, Robinson LJ, Mueller I, Felger I. Ultra-sensitive detection of Plasmodium falciparum by amplification of multi-copy subtelomeric targets. PLoS Med. 2015;2:e1001788.

4. Babiker HA, Gadalla AA, Ranford-Cartwright LC. The role of asymptomatic P. falciparum parasitaemia in the evolution of antimalarial drug resistance in areas of seasonal transmission. Drug Resist Updates. 2013;16:1-9.

5. WWARN Gametocyte Study Group. Gametocyte carriage in uncomplicated Plasmodium falciparum malaria following treatment with artemisinin combination therapy: a systematic review and meta-analysis of individual patient data. BMC Med. 2016;14:79.

6. Cheah PY, White NJ. Antimalarial mass drug administration: ethical considerations. Int Health. 2016:8:235-8.

7. Dierickx S, Gryseels C, Mwesigwa J, O’Neill S, Bannister-Tyrell M, Ronse M, et al. Factors associated with non-participation and non-adherence in directly observed mass drug administration for malaria in the Gambia. PLOS ONE. 2016;11:e0148627.

8. Wu Y, Ellis RD, Shaffer D, Fontes E, Malkin EM, Mahanty S, et al. Phase 1 trial of malaria transmission blocking vaccine candidates Pfs 25 and Pvs 25 formulated with montanide ISA 51. PLoS ONE. 2008:3:e2636.

9. Nikolaeva D, Draper SJ, Biswas S. Toward the development of effective transmission-blocking vaccines for malaria. Expert Rev Vaccines. 2015;14:653-80. 
10. Saxena AK, Singh K, Su HP, Klein MM, Stowers AW, Saul AJ, et al. The essential mosquito-stage P25 and P28 proteins from Plasmodium form tile-like triangular prisms. Nat Struct Mol Biol. 2006;13:90-1.

11. Sologub L, Kuehn A, Kern S, Przyborski J, Schillig R, Pradel G. Malaria proteases mediate inside-out egress of gametocytes from red blood cells following parasite transmission to the mosquito. Cell Microbiol. 2011;13:897-912.

12. Vermeulen AN, Roeffen WF, Henderik JB, Ponnudurai T, Beckers PJ, Meuwissen JH. Plasmodium falciparum transmission blocking monoclonal antibodies recognize monovalently expressed epitopes. Dev Biol Stand. 1985;62:91-7.

13. Tomas AM, Margos G, Dimopoulos G, van Lin LH, de Koning-Ward TF, Sinha R, et al. P25 and P28 proteins of the malaria ookinete surface have multiple and partially redundant functions. EMBO J. 2001;20:3975-83.

14. Baton $L A$, Ranford-Cartwright LC. Do malaria ookinete surface proteins P25 and P28 mediate parasite entry into mosquito midgut epithelial cells? Malar J. 2005;4:15.

15. Stura EA, Satterthwait AC, Calvo JC, Stefanko RS, Langeveld JP, Kaslow DC. Crystallization of an intact monoclonal antibody (4B7) against Plasmodium falciparum malaria with peptides from the Pfs 25 protein antigen. Acta Crystallogr D Biol Crystallogr. 1994;50:556-62.

16. Stowers AW, Keister DB, Muratova O, Kaslow DC. A region of Plasmodium falciparum antigen Pfs 25 that is the target of highly potent transmissionblocking antibodies. Infect Immun. 2000;68:5530-8.

17. van Amerongen A, Sauerwein RW, Beckers PJ, Meloen RH, Meuwissen $\mathrm{JH}$. Identification of a peptide sequence of the $25 \mathrm{kD}$ surface protein of Plasmodium falciparum recognized by transmission-blocking monoclonal antibodies: implications for synthetic vaccine development. Parasite Immunol. 1989;11:425-8.

18. Hartman ZC, Appledorn DM, Amalfitano A. Adenovirus vector induced innate immune responses: impact upon efficacy and toxicity in gene therapy and vaccine applications. Virus Res. 2008;132:1-14.

19. Li S, Locke E, Bruder J, Clarke D, Doolan DL, Havenga MJ, et al. Viral vectors for malaria vaccine development. Vaccine. 2007;25:2567-74.

20. Sakurai F, Kawabata K, Mizuguchi H. Adenovirus vectors composed of subgroup B adenoviruses. Curr Gene Ther. 2007;7:229-38.

21. Goodman AL, Blagborough AM, Biswas S, Wu Y, Hill AV, Sinden RE, et al. A viral vectored prime-boost immunization regime targeting the malaria Pfs 25 antigen induces transmission-blocking activity. PLOS ONE. 2011;6:e29428.

22. Li Y, Leneghan DB, Miura K, Nikolaeva D, Brian IJ, Dicks MD, et al. Enhancing immunogenicity and transmission-blocking activity of malaria vaccines by fusing Pfs 25 to IMX313 multimerization technology. Sci Rep. 2016;6:18848.

23. Wodrich $H$, Henaff D, Jammart B, Segura-Morales C, Seelmeir S, Coux $\mathrm{O}$, et al. A capsid-encoded PPXY-motif facilitates adenovirus entry. PLoS Pathog. 2010;6:e1000808

24. He TC, Zhou S, da Costa LT, Yu J, Kinzler KW, Vogelstein B. A simplified system for generating recombinant adenoviruses. Proc Natl Acad Sci USA. 1998;95:2509-14.

25. Luo J, Deng ZL, Luo X, Tang N, Song WX, Chen J, et al. A protocol for rapid generation of recombinant adenoviruses using the AdEasy system. Nat Protoc. 2007;2:1236-47.

26. Warming S, Costantino N, Court DL, Jenkins NA, Copeland NG. Simple and highly efficient BAC recombineering using galK selection. Nucleic Acids Res. 2005;33:e36.

27. Wiethoff CM, Wodrich H, Gerace L, Nemerow GR. Adenovirus protein VI mediates membrane disruption following capsid disassembly. J Virol. 2005;79:1992-2000
28. Saxena AK, Wu Y, Garboczi DN. Plasmodium p25 and p28 surface proteins: potential transmission-blocking vaccines. Eukaryot Cell. 2007;6:1260-5

29. Biasini M, Bienert S, Waterhouse A, Arnold K, Studer G, Schmidt T, et al. SWISS-MODEL: modelling protein tertiary and quaternary structure using evolutionary information. Nucleic Acids Res. 2014;42:W252-8.

30. Miura K, Deng B, Tullo G, Diouf A, Moretz SE, Locke E, Morin M, Fay MP, Long CA. Qualification of standard membrane-feeding assay with Plasmodium falciparum malaria and potential improvements for future assays. PLOS ONE. 2013:8:e57909.

31. Wu H, Han T, Belousova N, Krasnykh V, Kashentseva E, Dmitriev I, et al. Identification of sites in adenovirus hexon for foreign peptide incorporation. J Virol. 2005;79:3382-90.

32. Shiratsuchi T, Rai U, Krause A, Worgall S, Tsuji M. Replacing adenoviral vector HVR1 with a malaria B cell epitope improves immunogenicity and circumvents preexisting immunity to adenovirus in mice. J Clin Invest. 2010;120:3688-701.

33. Kubler-Kielb J, Majadly F, Wu Y, Narum DL, Guo C, Miller LH, et al. Longlasting and transmission-blocking activity of antibodies to Plasmodium falciparum elicited in mice by protein conjugates of Pfs25. Proc Natl Acad Sci USA. 2007;104:293-8.

34. Olive M, Eisenlohr L, Flomenberg N, Hsu S, Flomenberg P. The adenovirus capsid protein hexon contains a highly conserved human CD4+ T-cell epitope. Hum Gene Ther. 2002;13:1167-78.

35. Schuldt NJ, Amalfitano A. Malaria vaccines: focus on adenovirus based vectors. Vaccine. 2012;30:5191-8.

36. Wang C, Hart M, Chui C, Ajuogu A, Brian IJ, de Cassan SC, et al. Germinal center B cell and T follicular helper cell responses to viral vector and protein-in-adjuvant vaccines. J Immunol. 2016;197:1242-51.

37. McConnell MJ, Danthinne $X$, Imperiale MJ. Characterization of a permissive epitope insertion site in adenovirus hexon. J Virol. 2006;80:5361-70.

38. Miyata T, Harakuni T, Sugawa H, Sattabongkot J, Kato A, Tachibana M, et al. Adenovirus-vectored Plasmodium vivax ookinete surface protein, Pvs25, as a potential transmission-blocking vaccine. Vaccine. 2011;29:2720-6.

39. Worgall S, Krause A, Rivara M, Hee KK, Vintayen EV, Hackett NR, et al. Protection against $P$. aeruginosa with an adenovirus vector containing an OprF epitope in the capsid. J Clin Invest. 2005;115:1281-9.

40. Sharma B. Structure and mechanism of a transmission blocking vaccine candidate protein Pfs 25 from P. falciparum: a molecular modeling and docking study. In Silico Biol. 2008:8:193-206.

41. Saxena AK, Singh K, Long CA, Garboczi DN. Preparation, crystallization and preliminary $\mathrm{X}$-ray analysis of a complex between the Plasmodium vivax sexual stage $25 \mathrm{kDa}$ protein Pvs25 and a malaria transmissionblocking antibody Fab fragment. Acta Crystallogr D Biol Crystallogr. 2004;60:2054-7.

42. Saxena AK, Saul A, Garboczi DN. Crystallization and preliminary X-ray analysis of the Plasmodium vivax sexual stage 25 kDa protein Pvs25, a transmission-blocking vaccine candidate for malaria. Acta Crystallogr D Biol Crystallogr. 2004;60:706-8.

43. de Cassan SC, Forbes EK, Douglas AD, Milicic A, Singh B, Gupta P, et al. The requirement for potent adjuvants to enhance the immunogenicity and protective efficacy of protein vaccines can be overcome by prior immunization with a recombinant adenovirus. J Immunol. 2011;187:2602-16.

44. Emmer KL, Wieczorek L, Tuyishime S, Molnar S, Polonis VR, Ertl HC. Antibody responses to prime-boost vaccination with an HIV-1 gp145 Env protein and chimpanzee adenovirus vectors expressing HIV-1 gp140. Aids. 2016;16:2405-14. 